\title{
Um estudo bibliométrico da Estratégia do Oceano Azul
}

As empresas buscam maior lucratividade, e para isso se diferenciar dos concorrentes e buscar mercados com menor ou nenhuma concorrência se torna fundamental para o sucesso empresarial, neste contexto surge a Estratégia do Oceano Azul, onde a sua proposta é o aumento do valor e a redução de custos gerando inovação de valor. Desta forma este artigo tem como objetivo elucidar a atual relevância do tema e esclarecer os conceitos para facilitar a compreensão da Estratégia do Oceano Azul, explorando suas definições, ferramentas e princípios, auxiliando a procura por inovação de valor e mercados inexplorados. A metodologia utilizada foi a pesquisa bibliométrica nos portais Scopus, Emerald, Anpad e Scholar.Google. Percebe-se que nos primeiros cinco anos da publicação do livro "A Estratégia do Oceano Azul: como criar novos mercados e tornar a concorrência irrelevante", os resultados no Scholar.Google, apresentou a taxa média de crescimento de 53\%, para a expressão em inglês de 2006 à 2010, com 2588 resultados e nos cinco anos posteriores, de 2011 à 2015 tivemos 4.213 resultados. No portal Anpad apenas 3 publicações. Na base Emerald 21 publicações. E no portal Scopus tivemos 115 publicações demonstrando que o tema é relevante, ainda apresentando crescimento em produções acadêmicas, se recomenda para desenvolvimento futuro a realização de pesquisas em outras bases e comparação do resultado avaliando-se outras estratégias.

Palavras-chave: Estratégia do Oceano Azul; Inovação de Valor; Estudo Bibliométrico.

\section{A bibliometric study of the Blue Ocean Strategy}

Companies seek greater profitability, and that differentiate themselves from competitors and search for markets with less or no competition becomes critical to business success, in this context arises the Blue Ocean Strategy, where the proposal is to increase the value and the reduction of costs generating innovation value. This article aims to clarify the current relevance of the subject and clarify the concepts to facilitate the understanding of the Blue Ocean Strategy, exploring their definitions, tools and principles, helping the search for value innovation and untapped markets. The methodology used was the bibliometric research in Scopus, Emerald, Anpad and Scholar.Google. It is noticed that in the first five years of the publication of the book "Blue Ocean Strategy: How to Create new markets and make the competition irrelevant" considering the results of Scholar.Google, the average growth rate was 53\% for expression in English from 2006 to 2010 , with 2588 results and the five subsequent years, 2011 to 2015 we had 4,213 results. In Anpad portal only three publications. On Emerald 21 publications. Scopus portal had 115 publications demonstrating that the topic is relevant, still showing growth in academic productions. It is recommended for future development conducting research on other bases and comparing the result evaluating other strategies.

Keywords: Blue Ocean Strategy; Value Innovation; Bibliometric Study.

\section{Topic: Empreendedorismo}

Reviewed anonymously in the process of blind peer.

Felipe Ricardo Alencar Cattaneo

Universidade Potiguar, Brasil

http://lattes.cnpq.br/3465089905278060

felipe.cattaneo2010@gmail.com

\section{Lieda Amaral Souza}

Universidade do Minho, Portugal

http://lattes.cnpq.br/7703650553090691

lieda@dsi.uminho.pt

\section{Kleber Cavalcanti Nobrega}

Escola Politécnica da Universidade de São Paulo, Brasil

http://lattes.cnpq.br/7667731949775312

kleber@perceptum.com.br
Received: 20/01/2017

Approved: 20/03/2017
Referencing this:

CATTANEO, F. R. A.; SOUZA, L. A.; NOBREGA, K. C.. Um estudo bibliométrico da Estratégia do Oceano Azul. Revista Brasileira de Administração Científica, v.8, n.1, p.204-220, 2017. DOI: http://doi.org/10.6008/SPC2179-684X.2017.001.0016 


\section{INTRODUÇÃO}

Na busca por maior lucratividade, as empresas estão cada vez mais procurando um diferencial para sobrepujar os concorrentes e se estabelecer como líderes de mercado, sempre na procura de bons resultados no ambiente competitivo em que atuam. É preciso garantir a sustentabilidade da empresa com atuação focada, e inovar na busca de um diferencial que permita manter a atuação da empresa e o crescimento esperado para vencer as eventuais crises de mercado que estão presentes em qualquer seguimento. planejamento estratégico é o processo que proporciona sustentação para se manter na melhor direção atuando de forma inovadora e diferenciada (OLIVEIRA, 2013).

Mas às vezes, é preciso diversificar e buscar novos mercados, onde a briga por clientes não é tão acirrada. Neste sentido, muitas estratégias são utilizadas, mas acertar a estratégia não é tarefa simples, como afirma Mintzberg et al. (2000). A formação da estratégia envolve transformação e também perpetuação, onde a decisão estratégica é sempre orientada para o futuro e requer comprometimento (WRIGHT et al., 2009; MINTZBERG et al., 2000).

Porter (1980) teorizou sobre como as empresas devem prestar atenção na competição entre os concorrentes, levando em consideração cinco fatores ou forças competitivas que definem a concorrência entre elas, sendo estas: a ameaça de novos entrantes, o poder de negociação dos compradores, o poder de negociação dos fornecedores, a ameaça dos produtos ou serviços substitutos e a rivalidade entre as empresas existentes.

A rivalidade entre as empresas está presente intrinsicamente nos aspectos de definição de estratégias para superar os concorrentes, e para ficar acima dos demais, a empresa deve utilizar uma das três abordagens estratégicas genéricas: liderança no custo total, onde a empresa deve buscar a liderança de mercado pelo menor preço de custo total possível, seja por economia de escala, controle dos custos de produção ou qualquer outro meio que propicie ter menor custo que os concorrentes; diferenciação, onde a empresa deve buscar desenvolver produtos ou serviços únicos, seja por maior qualidade, desempenho ou características distintas dos produtos dos rivais; e enfoque, onde a empresa procura segmentos de mercados específicos, restritos, focando em determinados clientes, tipos de produtos ou região de atuação, utilizando a diferenciação e liderança em custos, ou ambas (PORTER, 1980).

Estudos sobre a competitividade das empresas, inovação de valor para se diferenciar no mercado e estratégias que busquem encontrar a melhor maneira da empresa sobressair entre os diversos concorrentes são presentes em literatura envolvendo administração estratégica.

Kim et al. (2005) traz à tona a perspectiva de não ser necessário trabalhar em um mercado saturado, mas sim buscar um novo mercado, onde os concorrentes mencionados por Porter (1980) não sejam mais importantes, pois os concorrentes seriam irrelevantes ou inexistentes, desta forma, podemos observar não um conflito aos conceitos de Porter, mas uma abordagem diferente ao se inovar em novas fronteiras de mercado buscando também clientes não explorados anteriormente, e após estudar 150 movimentos estratégicos em mais de cem anos e trinta setores econômicos, apresentam uma estratégia nomeada estratégia do oceano azul. 
O presente artigo discorre sobre teoria apresentada pelos autores W. Chan Kim e Renée Mauborgne no livro A Estratégia do Oceano Azul, lançado em 2005, onde são apresentados teoria e metodologia para busca de novos mercados não explorados, e onde a concorrência ainda é inexistente. Desta forma este artigo tem como objetivo elucidar a atual relevância do tema e esclarecer os conceitos para facilitar a compreensão da Estratégia do Oceano Azul, explorando suas definições, ferramentas e princípios, auxiliando a procura por inovação de valor e mercados inexplorados.

\section{REVISÃO TEÓRICA}

\section{Oceano Azul e Oceano Vermelho}

Kim et al. (2005) denomina de oceano vermelho os mercados atuais em que se encontram a maioria das empresas, buscando a cada momento conquistar os mesmos clientes com produtos parecidos e elevada concorrência gerando assim brigas pelo mercado que mancham o mercado, oceano, assim este mercado altamente competitivo. Já o mercado onde não existe concorrência pois um processo de inovação de valor com a redução dos custos gera um ambiente não concorrencial e desta forma não há conflitos, este mercado, é denominado de oceano azul. A figura 1 apresenta as diferenças entre as estratégias dos oceanos azul e vermelho (KIM et al., 2005).

\begin{tabular}{|l|l|}
\hline \multicolumn{1}{|c|}{ Estratégia do oceano vermelho } & \multicolumn{1}{c|}{ Estratégia do oceano azul } \\
\hline Competir nos espaços de mercado existentes. & Criar espaços de mercado inexplorados. \\
\hline Vencer os concorrentes. & Tornar a concorrência irrelevante. \\
\hline Aproveitar a demanda existente. & Criar e capturar a nova demanda. \\
\hline Exercer o trade-off valor-custo. & Romper o trade-off valor custo. \\
\hline $\begin{array}{l}\text { Alinhar todo o sistema de atividades da empresa com } \\
\text { sua escolha estratégica de diferenciação ou baixo } \\
\text { custo. }\end{array}$ & $\begin{array}{l}\text { Alinhar todo o sistema de atividades da empresa em } \\
\text { busca da diferenciação e baixo custo. }\end{array}$ \\
\hline $\begin{array}{l}\text { Figura 1. Estratégia do oceano vermelho versus estratégia do oceano azul } \\
\text { Fonte: Kim, W., \& Mauborgne, R. (2005). A estratégia do oceano azul: como criar novos mercados e tornar a concorrência } \\
\text { irrelevante. Rio de Janeiro: Elsevier. }\end{array}$
\end{tabular}

Figura 1: Estratégia do oceano vermelho versus estratégia do oceano azul. Fonte: Kim et al. (2005).

Esses oceanos azuis e vermelhos estão presentes em todos os segmentos. Um caso estudado no mercado de videogames mostrou que a Nintendo saiu do vermelho e navegou pelo azul, pelo menos por um tempo. Isso aconteceu após o lançamento de um controle de videogame no ano de 2006, em que os movimentos eram passados para a tela da televisão, a Nintendo com seu console de videogame chamado "Wii" atingiu o seu oceano azul, inovando não o equipamento de videogame com mais velocidade, ou qualidade de imagem, mas com uma nova forma de jogar.

Em sua nova forma de jogar, os pontos de destaque foram o controle de movimentos, forma de jogar única e orientação a família, abrangendo um grande público. Após a eliminação de Suporte a filmes, Redução da capacidade gráfica e física dos jogos, e a conectividade e armazenamento, elevaram a diversão e criaram a interatividade com o novo controle remoto e os personagens customizados por jogador. Ela permaneceu neste oceano até 2010 quando em setembro a empresa Sony lançou o 'Move' para uso no seu console 'Playstation 3' e em novembro do mesmo ano a Microsoft lançou o 'Kinect' para seu console 'X-Box 360', esses dois produtos seguiram na mesma linha de captura de movimento, imitando a maneira de jogar videogame que a Nintendo lançou como inovação quatro anos antes. 
Durante esse período, a Nintendo navegou em seu oceano azul mas foi encontrada neste mercado pelos seus concorrentes, a conclusão é que a estratégia do oceano azul não pode ser um processo estático, mas sim contínuo conforme artigo "O oceano azul que desapareceu - o caso do Nintendo Wii" (HOLLENSEN, 2013), e artigo “As melhores práticas de estratégia: o caso da Nintendo CO” (CRUZ JÚNIOR et al., 2008).

A criação desse mencionado 'oceano azul', um mercado onde a concorrência não afeta o negócio consiste em aumentar o valor para o comprador e na mesma proporção reduzir custos, esta inovação de valor é a principal característica para se obter a economia de custo, reduzindo a competição no setor, assim como aumentando o valor para os compradores com atributos nunca oferecidos anteriormente, conforme mostra a figura 2.

Enquanto nos mercados de ampla concorrência chamados de oceanos vermelhos, era preciso competir nos espaços que já são consolidados buscando vencer os concorrentes utilizando a demanda usual e utilizando as estratégias de diferenciação ou baixo custo, a teoria do oceano azul permite a criação de espaços não explorados, com concorrência irrelevante, graças a uma nova demanda com a utilização da diferenciação juntamente com o baixo custo, unindo as duas estratégias com os benefícios de cada uma delas, com uma chamada abordagem de visão reconstrucionista onde o alvo passa a ser criar novas regras de melhores práticas (KIM et al., 2005).

Stabell et al. (1998) tinha como proposta utilizar três tipos de valor: o valor da cadeia de suprimentos, onde se devia reduzir custos e oferecer aos clientes menores preços; o valor da loja, onde aspectos de resolver os problemas do cliente eram o foco; e o valor da network, onde o relacionamento entre clientes e fabricantes e também entre os próprios clientes fossem utilizados.

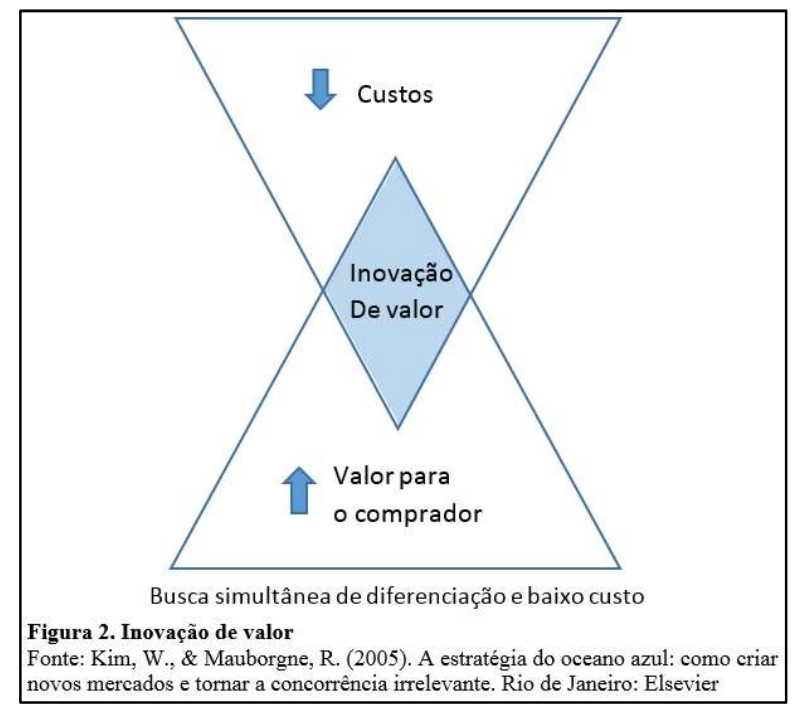

Figura 2: Inovação de valor. Fonte: Kim et al. (2005).

Apropriando-se das premissas deste artigo de 1998 e no modelo de três propostas de valor agregado, Sheehan et al. (2009) o artigo "Usando uma bússola de criação de valor para descoberta de "Oceanos Azuis". Nesse trabalho, Sheehan et al. (2009) propõe três tipos de valor para que as empresas encontrem o Oceano Azul através do aumento de valor ao produto, conforme autores, caso a empresa conseguisse atingir pelo menos dois dos três tipos seria possível chegar no mercado sem concorrências. 
Os três tipos são: Lógica da Eficiência Industrial, onde o valor agregado ao consumidor seria obtido com menor valor de produto graças a redução de custos de manufatura quando a empresa conseguisse atingir uma eficiência em sua linha de produção; Lógica dos serviços de Network, onde o relacionamento entre clientes, distribuidores, fabricantes podem agregar valor aos produtos e serviços, exemplos como os serviços de leilão do E-Bay, de namoro do website Match.com, leitores assíduos que influenciam na compra de outros produtos devido as suas opiniões em websites como Amazon.com, são colocados como exemplos para este item.

Por fim, Lógica de conhecimento intenso, onde existe aumento de valor agregado utilizando informações dos clientes para oferecer especificações únicas baseadas em seus gostos e desejos, de maneira simples seria guardar todas as informações possíveis dos clientes construindo um banco de dados para produzir produtos e oferecer serviços de acordo com os desejos dos clientes (STABELL et al., 1998; SHEEHAN et al., 2009).

\section{Princípios da Estratégia do Oceano Azul}

Seis princípios foram elaborados na estratégia do oceano azul, sendo quatro caracterizados como princípios de formulação e dois como princípios de execução. Princípios de formulação: Reconstrua as fronteiras do mercado; Concentre-se no panorama geral, e não nos números; Vá além da demanda existente; e Acerte a sequência estratégica. Os Princípios de execução são: Supere as principais barreiras organizacionais; e Introduza a execução na estratégia.

O primeiro princípio de formulação é (1) 'reconstrua as fronteiras do mercado', que traz a oportunidade de reduzir o risco da busca, identificando os caminhos utilizados para a criação de espaços inexplorados, e reduzindo ao máximo a concorrência tornando irrelevante a sua presença, basicamente realizando a avaliação e examinando os setores alternativos, os grupos estratégicos dentro dos setores, a cadeia de compradores, as ofertas de produtos e serviços complementares, os apelos funcionais e emocionais dos compradores e o transcurso do tempo.

Exemplo da aplicação deste princípio aconteceu na indústria de celulares que cresce a cada ano, milhares de aparelhos são vendidos todos os meses no mundo inteiro e parte destes aparelhos são fabricados na China, neste mercado tão competitivo a empresa MediaTek trabalha com fornecimento de 'chipset', equipamento eletrônico, para empresas de celular, e quando o governo Chinês liberou a manufatura de aparelhos de celulares em 2007 a empresa criou um novo modelo de negócio na fabricação de celular, auxiliando no mercado dos celulares sem marca, propiciando que pequenas empresas pudessem lançar seus celulares com peças fornecidas pela MediaTek, desta forma, a empresa e seus parceiros encontraram um mercado inexplorado de quem procura um aparelho com as mesmas funcionalidades das grandes marcas, mas sem o alto valor da marca (CHANG, 2010).

O segundo princípio é (2) 'Concentre-se no panorama geral, não nos números', onde objetivamos reduzir o risco do planejamento desenhando a matriz de avaliação de valor aplicando o processo dos quatro 
passos para visualização da estratégia conforme descrito na figura 3, utiliza-se uma abordagem visual para construir uma estratégia, criando e explorando oportunidades não aproveitadas (KIM et al., 2005).

\begin{tabular}{|c|c|c|c|}
\hline Despertar Visual & Exploração Visual & $\begin{array}{c}\text { Feira de Estratégia } \\
\text { visual }\end{array}$ & Comunicação Visual \\
\hline 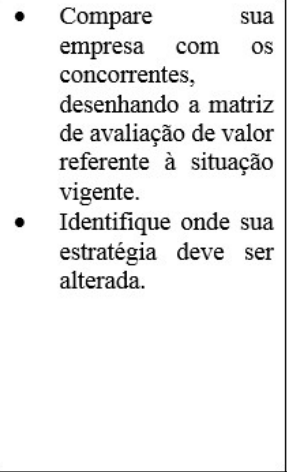 & $\begin{array}{l}\text { - Saia do campo para } \\
\text { explorar as seis } \\
\text { fronteiras que levam } \\
\text { à criação de oceanos } \\
\text { azuis. } \\
\text { Observe as } \\
\text { vantagens } \\
\text { diferenciadoras de } \\
\text { produtos e serviços } \\
\text { alternativos. } \\
\text { Veja que fatores se } \\
\text { devem eliminar, } \\
\text { criar ou mudar. }\end{array}$ & $\begin{array}{l}\text { - Desenhe a sua futura } \\
\text { matriz, com base em } \\
\text { insights resultantes } \\
\text { das observações de } \\
\text { campo. } \\
\text { Receba feedback } \\
\text { sobre a matriz de } \\
\text { avaliação de valor de } \\
\text { alternativas, oriundo } \\
\text { de clientes, clientes } \\
\text { dos concorrentes e } \\
\text { não clientes. } \\
\text { Use o feedback na } \\
\text { construção da } \\
\text { melhor estratégia } \\
\text { para o futuro. }\end{array}$ & $\begin{array}{l}\text { - Distribua os seus } \\
\text { perfis estratégicos } \\
\text { iniciais e finais numa } \\
\text { única página para } \\
\text { fácil comparação. } \\
\text { - Apoie apenas os } \\
\text { projetos e iniciativas } \\
\text { que permitam à sua } \\
\text { empresa fechar as } \\
\text { lacunas na } \\
\text { implementação da } \\
\text { nova estratégia. }\end{array}$ \\
\hline
\end{tabular}

Figura 3: Os quatro passos para a visualização da estratégia. Fonte: Kim et al. (2005)

O princípio de número três é (3) 'Vá além da demanda existente' o que reduz o risco de escala associado à criação de novos mercados com a busca de clientes que não estão no seu próprio mercado, a teoria destaca três níveis de não clientes que podem se tornar clientes, conforme figura 4, os chamados 'quase convertidos' são aqueles clientes que estão no seu mercado mas ainda não compram seu produto, os clientes 'refratários' que não compram seu produto por escolha própria, e os clientes 'inexplorados' que estão fora do atual mercado de atuação de sua empresa, são clientes que não foram imaginados como clientes para o seu produto (KIM et al., 2005).

Neste modelo de buscar clientes não imaginados, em 2010 a estratégia do oceano azul e este princípio foram aplicados para identificar um novo mercado inexplorado para frutas e verduras na Europa, a técnica proposta foi utilizada para encontrar nas crianças e adolescentes o mercado de fruta jovem, onde frutas frescas e adocicadas foram embaladas como 'doces', em tamanhos adaptados, desta forma, como um alimento saudável, o que tem forte apelo atualmente, o alvo de crianças e adolescentes surtiu efeito. Entre os fatores de destaque da matriz de avaliação de valor estão o tamanho adequado da fruta, embalagem atrativa, novos pontos de vendas e preço por pedaço (WUBBEN et al., 2012).

O quarto princípio é (4) 'Acerte a sequência estratégica' onde se reduz o risco do modelo de negócio elaborando a estratégia do oceano azul, considerando os principais critérios que são: 'adoção', 'custo', 'preço' e 'utilidade para o comprador'. Com a resposta a algumas perguntas é possível verificar se a estratégia do oceano azul possui viabilidade comercial, conforme consta na figura 5. Cada uma das perguntas é feita de maneira que se a resposta for sim pode-se seguir adiante, caso contrário será necessário repensar e reiniciar a avaliação.

A pergunta para verificar a utilidade para o comprador é: 'A sua ideia de negócios vai gerar utilidade excepcional ao comprador?'. É preciso uma resposta afirmativa para seguir para a pergunta que remete ao preço do produto ou serviço: 'O seu preço é facilmente acessível para a massa de compradores?'. Sabemos que a estratégia tem como base aumentar o valor agregado para o comprador, sendo necessário um preço 
razoável para o produto, assim, somente com um preço razoável é possível prosseguir para a pergunta que remete ao custo: 'Você será capaz de cumprir sua meta de custo para que possa lucrar ao preço estratégico?'.

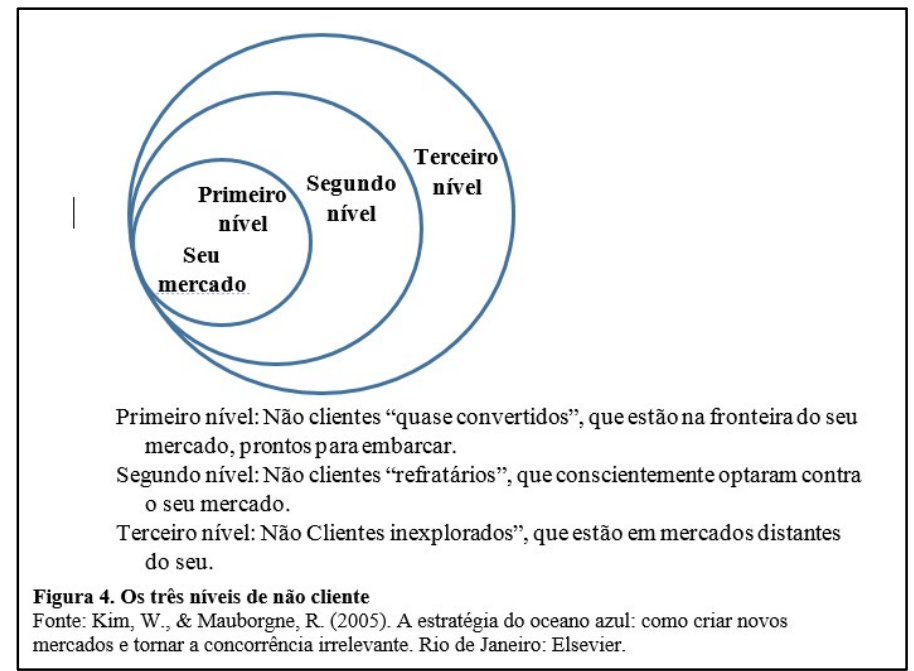

Figura 4: os três níveis de não cliente. Fonte: Kim et al. (2005)

Um baixo custo e um preço acessível aliado a uma utilidade para o comprador direcionam para a última pergunta referente a adoção, 'Quais são as barreiras na adoção para que você realize sua ideia de negócios? Você as está encarando?', ou seja, é possível ultrapassar as barreiras cognitivas, de recursos, motivacional e políticas? a empresa deve encarar todas as barreiras encontradas, enfrenta-las e supera-las, caso seja possível, pode-se considerar a estratégia do oceano azul comercialmente viável, mas qualquer resposta negativa a qualquer uma destas perguntas, remete para a reavaliação dos atributos envolvidos na pergunta anterior para que se consiga seguir na correta sequência da estratégia proposta.

Esses quatro primeiros princípios são os chamados princípios de formulação, pois trabalham na formulação das premissas básicas referenciais que as empresas deverão tratar durante o início do processo de migração para um mercado sem concorrências e com alvo valor agregado nos produtos oferecidos aos seus clientes (KIM et al., 2005).

Os dois últimos princípios são os chamados princípios de execução da estratégia, o quinto (5) 'Supere as principais barreiras organizacionais' que trata de da superação de quatro barreiras, que se colocam frente à execução da estratégia, a primeira a 'barreira cognitiva', que cega os empregados para a necessidade imperiosa da mudança radical, em segundo a 'barreira dos recursos', que é endêmica nas empresas, a busca por recursos que permitem crescimento, em terceiro a 'barreira motivacional', que desestimula e desmoraliza o pessoal, e por último, a 'barreira política' da resistência externa e interna à mudança.

O sexto princípio trazido por Kim et al. (2005) é 'Introduza a execução na estratégia', onde é importante que todos os funcionários entendam e compreendam a estratégia da empresa, e somente com o alinhamento entre todos os níveis hierárquicos a superação dos obstáculos organizacionais serão vencidos, para isso é necessário o que se denominou de processo justo, onde a cooperação voluntária impera levando todos na mesma direção na execução das decisões estratégicas.

Para isso, são necessárias três características: envolvimento, ou seja engajamento e comprometimento de todos os funcionários da empresa independente do cargo ou nível hierárquico; 
explicação, na qual todos devem conhecer as estratégias, suas razões e os seus critérios; e clareza das expectativas, o que se espera de cada um dos envolvidos no processo e da empresa, quais os objetivos a serem alcançados, o que cada funcionário deve fazer para garantir que as expectativas sejam atendidas (KIM et al., 2005).

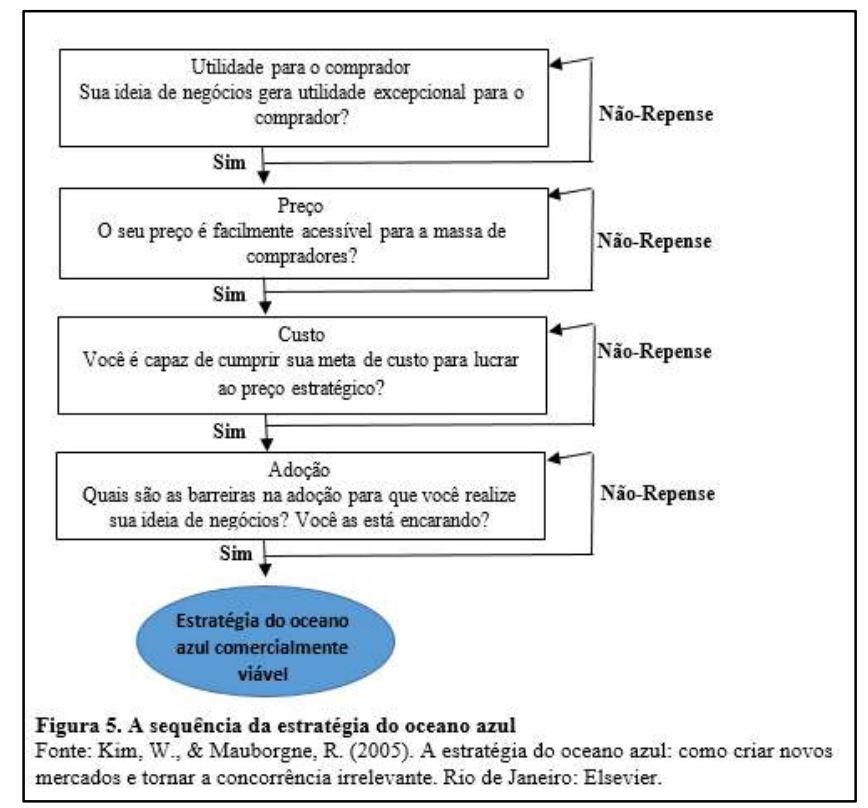

Figura 5: A sequência da estratégia do oceano azul. Fonte: Kim et al. (2005).

Seguindo esses dois princípios, um artigo de Lindic et al. (2012), buscou atrelar a política econômica das empresas com a estratégia do oceano azul investigando o rápido crescimento. No estudo, foram consideradas quatro premissas: "criar um novo espaço de mercado leva a um crescimento acelerado", onde ficou confirmado que as primeiras empresas a explorar estes mercados crescem mais rápidas, não basta apenas criar; "empresas de rápido crescimento podem ser encontradas em diversos nichos", o que também ficou confirmado; "empresas crescem rapidamente com inovação pioneira de valor e não somente com inovação pioneira de tecnologia", e se confirmou que a chave está na inovação de valor e não somente na inovação tecnológica; e o crescimento acelerado independe do tamanho da empresa, o que também ficou comprovado neste artigo (LINDIC et al., 2012).

\section{Instrumentos para definição da Estratégia do Oceano Azul}

Para conseguir um modelo de estratégia de oceano azul, Kim et al. (2005) desenvolveu instrumentos e modelos a serem utilizados em seis princípios definidos na estratégia. O primeiro é a matriz de avaliação de valor que tem como propósito captar a situação atual no mercado de atuação já conhecido e o que os compradores recebem como clientes das ofertas de mercado, são elencados para cada tipo de setor os atributos nos quais se baseiam a competição seja em produtos, serviços e entrega, basicamente expondo onde o setor mais investe e compete. O segundo instrumento é chamado de modelo das quatro ações, baseado em quatro perguntas-chave usadas para se redefinir os atributos da matriz de avaliação de valor, representado na figura 6.

Utilizando o modelo de quatro ações e a inovação tecnológica foi identificado que o operador logístico CJ - Global Logistics Service (CJ-GLS) que iniciou como subsidiário da Samsung de tecnologia e 
sistemas logísticos, e logo depois separou com abertura de capital se tornando empresa independente atingiu seu oceano azul, expandindo as suas capacidades de atuação, com a ajuda de inovação tecnológica, recursos humanos e infraestrutura (KIM et al., 2008).

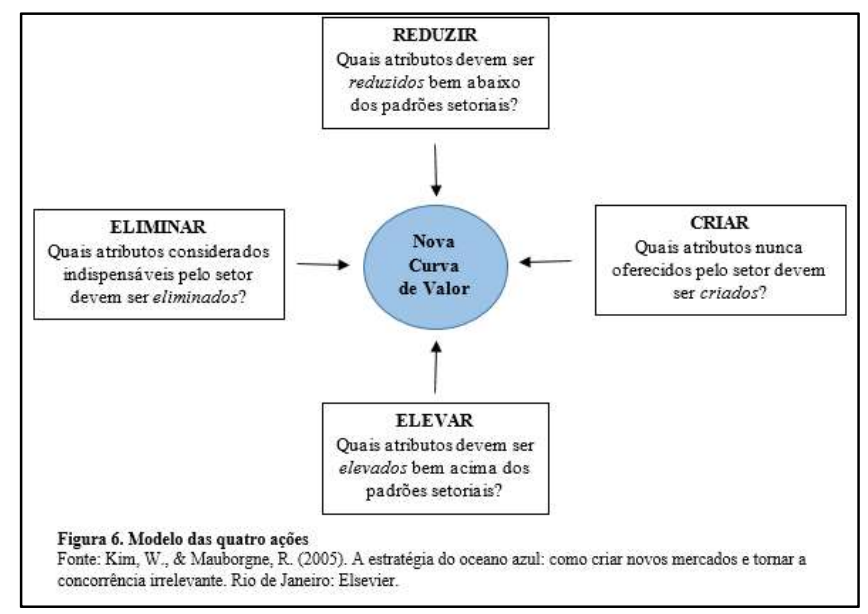

Figura 6: Modelo das quatro ações. Fonte: Kim et al. (2005).

O terceiro instrumento é a chamada matriz eliminar-reduzir-elevar-criar onde se colocam os atributos em cada uma das quatro ações, conforme as respostas para as perguntas do modelo proposto, desta forma, ficam mapeados os atributos que vão ser utilizados para a criação de uma nova curva de valor. Esta nova curva de valor deverá apresentar três características da boa estratégia, são elas: foco, singularidade e mensagem consistente (KIM et al., 2005).

Utilizando esta matriz foi realizada pesquisa pratica com 32 gerentes de 15 hotéis em Taiwan na busca dos atributos essenciais para chegar ao oceano azul no mercado hoteleiro na cidade, o estudo concluiu que os preços, produtos e estratégias de marketing relevantes que foram implementadas nos hotéis estudados enfatizam os conceitos de valor e inovação de um oceano azul (YANG, 2012). A utilização dos instrumentos propostos nesta nova teoria pode ser observada em Carvalho (2014) e João (2011) com o uso do modelo de quatro ações, e também em Oliveira (2013) no desenvolvimento de um novo produto de software.

\section{METODOLOGIA}

O presente estudo utilizou o método de pesquisa bibliométrica. No dia 16 de Março de 2016 foram realizadas buscas através das palavras-chave: estratégias do oceano azul, e tem como base uma revisão de literatura da teoria apresentada pelos autores W. Chan Kim e Renée Mauborgne no livro 'A Estratégia do Oceano Azul: como criar novos mercados e tornar a concorrência irrelevante', livro lançado em 2005, que já possui mais de 3,5 milhões de exemplares vendidos em mais de 43 idiomas e está sendo lançado em edição estendida neste ano de 2015 (PUBLISHNEWS, 2015). Buscou-se esclarecer e exemplificar, as considerações do uso da metodologia apresentada no livro, apresentando uso real dos conceitos, ferramentas e princípios da teoria apresentada neste artigo.

Para contribuir com a avaliação sobre a atual relevância do tema foram levantados os dados através de consulta ao website 'www.scholar.google.com' e 'www.scholar.google.com.br', onde foram classificados 
os números de resultados encontrados para a pesquisa por: 'Blue Ocean Strategy', e também pela expressão 'Estratégia do Oceano Azul', com a utilização de aspas, lembrando que as pesquisas foram realizadas sem as marcações de 'incluir patentes' e 'incluir citações', e para a obtenção do período foi especificado o intervalo para cada ano, desta forma foi possível obter o resultado anual, por exemplo: 2005 - 2005, e assim por diante.

Também foi verificado a presença das expressões no mecanismo de busca na biblioteca eletrônica de periódicos científicos da Anpad, busca no portal Scopus, uma das maiores bases de dados de resumos e literatura científica e também na base de pesquisas Emerald, uma base de consulta de periódicos com concentração nas áreas de administração contabilidade, finanças e economia, conforme as etapas do processo de pesquisa referenciada no quadro 1.

Quadro 1: Etapas do processo de pesquisa bibliométrica.

\begin{tabular}{|l|l|l|}
\hline Etapa & \multicolumn{1}{|c|}{ Ação 1 } & \multicolumn{1}{|c|}{ Ação 2 } \\
\hline 1 & $\begin{array}{l}\text { Busca no Scholar.Google } \\
\text { Expressão: Estratégia Oceano Azul e Blue Ocean Strategy }\end{array}$ & Ordenar por ano e avaliar resultados \\
\hline 2 & $\begin{array}{l}\text { Busca no portal Anpad } \\
\text { Expressão: Estratégia Oceano Azul e Blue Ocean Strategy }\end{array}$ & Avaliação dos títulos apresentados \\
\hline 3 & $\begin{array}{l}\text { Busca no portal Scopus } \\
\text { [Article Title, Abstract, Keywords: “strategy"] AND [Article Title, } \\
\text { Abstract, Keywords: “blue ocean"] }\end{array}$ & $\begin{array}{l}\text { Ordenar os resultados por ano de publicação, } \\
\text { país, tipo de documento, tipo de fonte, área } \\
\text { de pesquisa e palavras chaves }\end{array}$ \\
\hline 4 & $\begin{array}{l}\text { Busca na base Emerald } \\
\text { [Abstract: blue ocean strategy] OR } \\
\text { [Publication Title: blue ocean strategy] }\end{array}$ & $\begin{array}{l}\text { Ordenar os resultados por ano de publicação, } \\
\text { tipo de documento, área de pesquisa e } \\
\text { periódico }\end{array}$ \\
\hline
\end{tabular}

\section{RESULTADOS E DISCUSSÃO}

Em uma recente entrevista realizada em 2015 pela revista Strategy \& Leadership, o editor Robert M Randall esclarece com os autores Kim e Mauborgne alguns dos principais mitos referentes a estratégia do oceano azul e outras abordagens de inovação e estratégias de marketing (RANDALL, 2015). Kim e Mauborgne esclarecem que é importante focar não nos clientes usuais mas sim em "não clientes" ou também conhecidos como 'clientes inexplorados', para se criar uma nova demanda, este é um dos grandes destaques da estratégia, prova disto pode ser observado no mercado de frutas para jovens e crianças na Europa (WUBBEN et al., 2012).

$\mathrm{Na}$ entrevista esclarecem que a empresa não precisa necessariamente sair de seu ramo de atividade, como fica comprovado pela empresa MediaTek, que apenas expandiu as barreiras de seus clientes para outras fábricas de celulares (CHANG, 2010). O mito que de a empresa precisa de inovação tecnológica, caso como da CJ-GLS onde a melhoria da operação logística foi determinante (KIM et al., 2012), e corroboram para reforçar o mito, mas os autores da teoria destacam que apesar da inovação tecnológica ser importante e ajudar no processo, apenas com a inovação de valor é que se atinge o oceano azul, a tecnologia pode em produtos e serviços facilitar, agradar ou divertir os compradores, mas são estes atributos qualitativos que definem a inovação de valor e não a tecnologia em si.

Sobre o mito de que para se sustentar no oceano azul a empresa deve ser a primeira a entrar no mercado, Kim e Mauborgne afirmam que o importante não é chegar primeiro, mas sim ser a primeira empresa neste mercado a agregar a inovação ao valor para o consumidor, o que se comprova com o caso 
estudado da Amazom.com que não foi a primeira empresa a entrar no mercado de comércio eletrônico, e não foi a primeira empresa a vender livros pela internet, mas seu crescimento e estabilidade lhe coloca em um oceano azul que já existia (SHEEHAN et al., 2009).

Outro mito abordado na entrevista foi que a estratégia do oceano azul é sinônimo de estratégia da diferenciação, ou sinônimo de estratégia de baixo preço de custo e baixo preço de venda, os autores lembram que a estratégia da diferenciação utiliza o alto preço de custo e alto preço de venda para agregar valor ao cliente, e no caso do oceano azul a ideia é que se use a diferenciação e o baixo custo simultaneamente, mas não apenas com baixo preço, mas sim com um preço estratégico, e que seja de alto valor agregado ao cliente, podemos observar isso acontecer no caso da Nintendo, onde o console "Wii" veio trazer um videogame com preço próximo aos que eram praticados no mercado, mas com um atrativo e inovação de valor agregado em seu controle de captura de movimentos (CRUZ JÚNIOR et al., 2008; HOLLENSEN, 2013; RANDALL, 2015).

\section{Scholar Google}

Os dados obtidos através da consulta na internet pelas expressões 'Blue Ocean Strategy' e pela expressão 'Estratégia do Oceano azul' estão apresentados na tabela 1, e mostram a quantidade de resultados exibidos para a consulta ano após ano, nos últimos dez anos, desde 2005 até o ano de 2015, com a diferença incremental para cada ano analisado, e o percentual de diferença em relação ao ano anterior, além de um resultado de taxa média de crescimento nos dois quinquênios analisados.

Percebe-se que nos primeiros cinco anos da publicação do livro, a taxa média de crescimento foi de 53\%, para a expressão em inglês de 2006 à 2010, com 2.588 resultados, e nos cinco anos posteriores, de 2011 à 2015 apesar da taxa média de crescimento apresentar o valor de 2,1\%, um declínio na curva de crescimento, demonstrando que o assunto cresceu menos que no período anterior, ainda se apresentam 4.213 resultados, $63 \%$ mais resultados que o quinquênio anterior.

Tabela 1: Resultados no scholar.google.

\begin{tabular}{|c|c|c|c|c|c|c|c|c|c|c|c|}
\hline \multicolumn{12}{|c|}{ Quantidade de resultados na consulta por: "Blue Ocean Strategy" no Scholar.google.com } \\
\hline Ano & 2005 & 2006 & 2007 & 2008 & 2009 & 2010 & 2011 & 2012 & 2013 & 2014 & 2015 \\
\hline Quantidade de Resultados & 110 & 274 & 406 & 436 & 635 & 727 & 797 & 883 & 880 & 857 & 796 \\
\hline Aumento em relação ao ano anterior & - & 164 & 132 & 30 & 199 & 92 & 70 & 86 & -3 & -23 & -61 \\
\hline Aumento \% em relação ao ano anterior & - & $149,1 \%$ & $48,2 \%$ & $7,4 \%$ & $45,6 \%$ & $14,5 \%$ & $9,6 \%$ & $10,8 \%$ & $-0,3 \%$ & $-2,6 \%$ & $-7,1 \%$ \\
\hline Média do Quinquênio para o aumento \% & & \multicolumn{5}{|c|}{$53,0 \%$} & \multicolumn{5}{|c|}{$2,1 \%$} \\
\hline \multicolumn{12}{|c|}{ Quantidade de resultados na consulta por: "Estratégia do Oceano Azul" no Scholar.google.com.br } \\
\hline Ano & 2005 & 2006 & 2007 & 2008 & 2009 & 2010 & 2011 & 2012 & 2013 & 2014 & 2015 \\
\hline Quantidade de Resultados & 1 & 7 & 18 & 21 & 40 & 28 & 46 & 47 & 63 & 67 & 67 \\
\hline Aumento em relação ao ano anterior & - & 6 & 11 & 3 & 19 & -12 & 18 & 1 & 16 & 4 & 0 \\
\hline Aumento \% em relação ao ano anterior & - & $600,0 \%$ & $157,1 \%$ & $16,7 \%$ & $90,5 \%$ & $-30,0 \%$ & $64,3 \%$ & $2,2 \%$ & $34,0 \%$ & $6,3 \%$ & $0,0 \%$ \\
\hline Média do Quinquênio para o aumento \% & & \multicolumn{5}{|c|}{$166,9 \%$} & \multicolumn{5}{|c|}{$21,4 \%$} \\
\hline
\end{tabular}

Para a expressão em português observa-se que nos primeiros cinco anos a taxa média de crescimento para os resultados obtidos foi de 166,9\%, muito acima do resultado encontrado para o mesmo período analisando-se a expressão em inglês, isso mostra que em português nos primeiros cinco anos o crescimento na relevância do assunto foi maior, apesar de apenas 115 resultados, mas destaca-se que no segundo período de cinco anos, de 2011 à 2015 apesar da taxa de crescimento de apenas $21,4 \%$, os 290 resultados representam $152 \%$ de aumento em relação ao quinquênio anterior, o mesmo efeito de maior número absoluto apresentado com a expressão em inglês para os últimos 5 anos. Na análise de distribuição temporal, o número de resultados nos últimos anos se mantém elevado como pode ser observado na figura 7. 


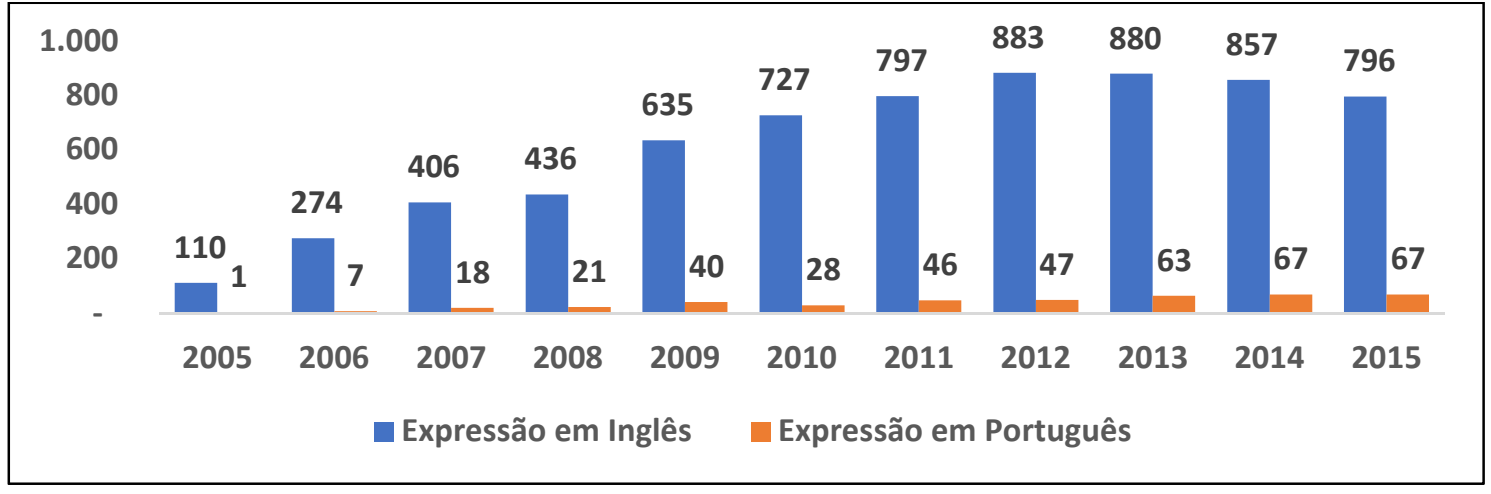

Figura 7: Resultados do site 'scholar.google' para as expressões em inglês e português.

\section{Anpad}

A busca pelas expressões no portal de pesquisas da Anpad através do portal 'http://www.spell.org.br/' apresentou apenas três artigos, um publicado em 2007 'Posicionamento da marca-país Brasil: uma proposta de estratégia do oceano azul' (GALHANONE et al., 2007), outro artigo publicado em 2010 "Reflections on Blue Ocean Strategy: a comparison with Ansoff's, Porter's, and Hax and Wilde's strategies" (JUSSANI et al., 2010), e um publicado em 2015 "Estratégia do Oceano Azul: Relato de Implantação em um Setor em Crise” (BARBERO et al., 2015).

\section{Emerald}

Na base de pesquisa 'Emerald' foram encontrados 21 resultados distribuídos anualmente conforme gráfico 8 considerando o período compreendido entre 2004 e 2016, pode ser constatado que o número de publicações teve um pico em 2012, mas com apenas 4 publicações no período de 2013 à 2015 . Os tipos de documentos que mais foram presentes são os Artigos Conceituais e os Artigos de Pesquisa cada um com 6 publicações, seguidos pelas Revisões e Estudos de Caso com 3 publicações cada, como pode ser observado na figura 9.

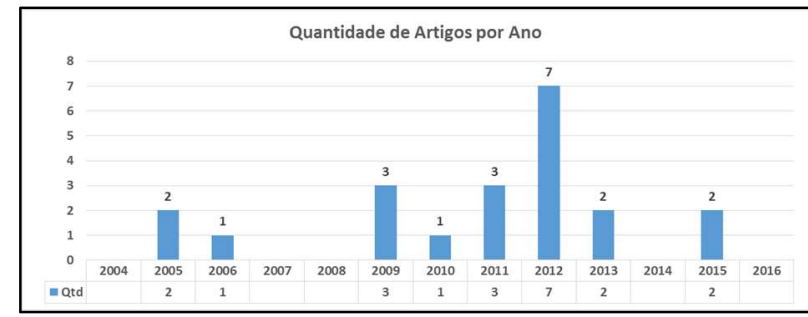

Figura 8: Resultados de pesquisa na base Emerald Artigos por Ano de Publicação.

\begin{tabular}{|l|c|}
\hline \multicolumn{1}{|c|}{ Tipos de Documento } & Quantidade \\
\hline Artigo Conceitual (Conceptual paper) & 6 \\
\hline Artigo de Pesquisa (Research paper) & 6 \\
\hline Revisão Geral (General review) & 3 \\
\hline Estudo de Caso (Case study) & 3 \\
\hline Artigo secundário (Secondary article) & 2 \\
\hline Ponto de vista (Viewpoint) & 1 \\
\hline
\end{tabular}

Figura 9: Resultados de pesquisa na base Emerald Tipos de Documento.

Os periódicos que mais publicaram artigo foram 'Strategy \& Leadership' com apenas 04 publicações e 'Journal of Business Strategy' com 03 publicações, conforme figura 10. As áreas temáticas as quais as publicações estavam relacionadas estão descritas na figura 11, onde se destaca o tema Estratégia. Foram identificadas 40 palavras chaves constantes nas publicações e a figura 12 demonstra as que mais aparecem nos textos encontrados na base de pesquisa Emerald. 


\begin{tabular}{|c|l|}
\hline Quantidade & \multicolumn{1}{|c|}{ Periódico } \\
\hline 4 & Strategy \& Leadership \\
\hline 3 & Journal of Business Strategy \\
\hline 2 & Jnl of Product \& Brand Mgt, Journal of Strategy and Mgt, Strategic Direction \\
\hline 1 & $\begin{array}{l}\text { Asia-Pacific J of Bus Admin, British Food Journal, Corporate Governance, Innovation Science, Int J Contemp Hospitality } \\
\text { Mngt, Management Decision, Mrkting Intelligence \& Plan, Emerging Markets Case Studies Collection }\end{array}$ \\
\hline
\end{tabular}

Figura 10: Resultados de pesquisa na base Emerald - Periódico.

\begin{tabular}{|c|l|}
\hline Quantidade & \multicolumn{1}{|c|}{ Área } \\
\hline 11 & Estratégia \\
\hline 8 & Estratégia Corporativa \\
\hline 6 & Estratégia Competitiva \\
\hline 4 & Liderança \\
\hline 3 & Marketing \\
\hline 2 & $\begin{array}{l}\text { Equidade/Gestão da Marca, Ciências empresariais e Operações, Ciências empresariais/Pesquisa operacional, Preço, } \\
\text { Gestão de produtos e Gestão estratégica/Planejamento }\end{array}$ \\
\hline 1 & $\begin{array}{l}\text { Imagem da Marca no Turismo, Governança corporativa, Gestão de destinos/marketing, Gestão de comida e bebida, } \\
\text { Alimentos e nutrição, Gestão hoteleira, Historia da Gestão/Teoria, Ciência de Marketing, Turismo e Hospitalidade }\end{array}$ \\
\hline
\end{tabular}

Figura 11: Resultados de pesquisa na base Emerald - Área.

\begin{tabular}{|c|l|}
\hline Quantidade de aparições & \multicolumn{1}{c|}{ Palavras Chave } \\
\hline 7 & Blue Ocean Strategy \\
\hline 4 & Competitive strategy, Innovation \\
\hline 3 & Management strategy, Strategic planning \\
\hline 2 & $\begin{array}{l}\text { Customer orientation, Customer relationship management, Hospitality } \\
\text { management, Product development, Taiwan, Value innovation }\end{array}$ \\
\hline 1 & $\begin{array}{l}\text { Asset valuation, Best value, Blue ocean, BOS, Business analysis, Business } \\
\text { excellence, Business model transformation }\end{array}$ \\
\hline
\end{tabular}

Figura 12: Resultados de pesquisa na base Emerald - Palavras Chave.

\section{Scopus}

No portal Scopus foi realizada a pesquisa utilizando a pesquisa avançada e buscando pela palavra 'estratégia' (Strategy) considerando a inclusão em um dos títulos dos artigos, resumos e palavras chaves, e também (And) as palavras 'oceano azul' (Blue Ocean) também considerando a inclusão nos títulos dos artigos, resumos e palavras chave, em todos os tipos de documento, todos os períodos e todas as áreas. Foram encontradas 115 publicações no período compreendido entre 2004 e 2016, distribuídos anualmente conforme figura 13.

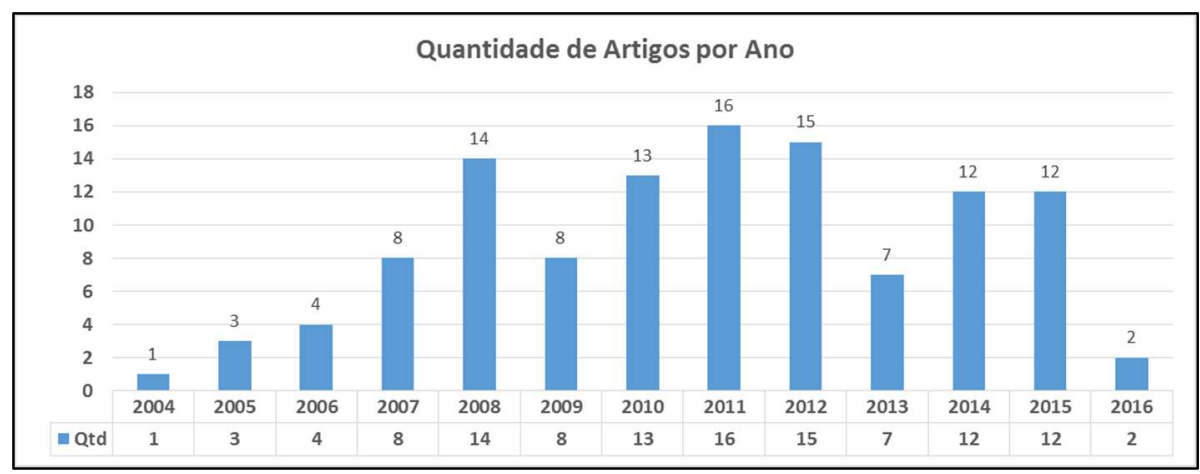

Figura 13: Resultados de pesquisa na Scopus - Ano de Publicação.

A figura 14 apresenta, dos 8 tipos de documentos referentes as publicações na Scopus, 51,2\% (64) das publicações são artigos acadêmicos e 29\% (33) são artigos apresentados em congressos e conferências, evidenciando o caráter científico do tema. De forma conceitual, a figura 14 apresenta os resultados de pesquisa na Scopus por tipo de documento. 


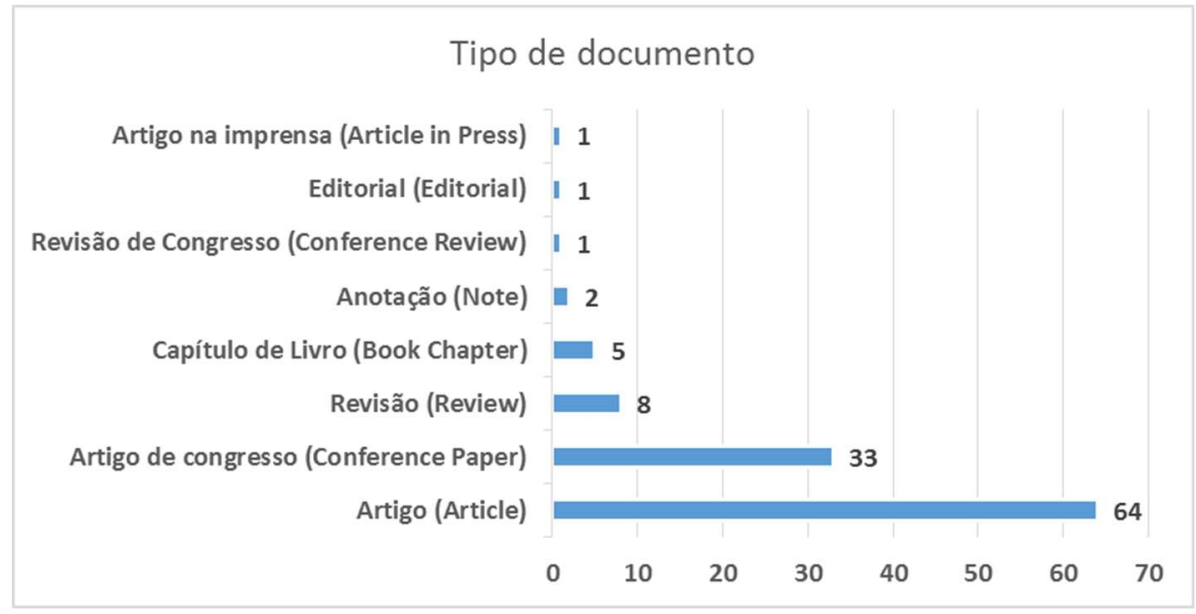

Figura 14: Resultados de pesquisa na Scopus - Tipo de Documento.

As publicações se apresentam em 35 países, isso mostra a abrangência geográfica que o tema possui, onde os países com maior número de publicações são os Estados Unidos com 24, Taiwan com 13, China e Itália com 7 publicações cada, a figura 15 detalha todos os demais países, referindo-se aos resultados da base de pesquisa Scopus.

\begin{tabular}{|c|l|}
\hline Quantidade & \multicolumn{1}{|c|}{ País } \\
\hline 24 & Estados Unidos \\
\hline 13 & Taiwan \\
\hline 7 & China e Itália \\
\hline 6 & Coréia do Sul e Malásia \\
\hline 4 & Espanha \\
\hline 3 & Brasil, Canadá, Finlândia, França, Alemanha, Hong Kong, Holanda, México e Reino Unido \\
\hline 2 & India, Irã, Portugal e África do Sul \\
\hline 1 & $\begin{array}{l}\text { Belgica, Chipre, República Checa, Dinamarca, Irlanda, Omã, Polônia, Romênia, Rússia, } \\
\text { Eslováquia, Eslovênia, Suécia, Macau, Tailândia e Austrália }\end{array}$ \\
\hline
\end{tabular}

Figura 15: Resultados de pesquisa na Scopus - País.

O estudo separou as publicações por 21 áreas temáticas de influência, conforme encontrado na base Scopus e se destacou a área de Administração, Negócios e Contabilidade, que apresentou 55 publicações, 48\% do total de 115, nas demais 20 áreas destacam-se Engenharia com 25 publicações e Ciências da Computação com 19, a quantidade em detalhe das demais áreas podem ser observadas na figura 16.

\begin{tabular}{|c|l|}
\hline Quantidade & \multicolumn{1}{|c|}{ Área } \\
\hline 55 & Administração, Negócios e Contabilidade \\
\hline 25 & Engenharia \\
\hline 19 & Ciências da Computação \\
\hline 16 & Ciências Sociais \\
\hline 15 & Ciências de Decisão \\
\hline 14 & Economia, Econometria e Finanças \\
\hline 6 & Ciências biológicas e agrícolas \\
\hline 4 & Artes e Humanas, Ciências dos Materiais e Medicina \\
\hline 2 & Bioquímica, Genetica e Biologia Molecular, Ciências ambientais, Farmacologia, Toxicologia e Farmáceutica, Psicologia e Veterinária \\
\hline 1 & Engenharia Química, Ciências Planetárias e da Terra, Profissionais de Saúde, Matemática, Multidisciplinar e Enfermagem \\
\hline
\end{tabular}

Figura 16: Resultados de pesquisa na Scopus - Área.

Nas 115 publicações pesquisadas, existem 438 palavras chaves, mas com pouca repetição de vocábulo. Temos 39 'Blue Ocean Strategy' (Estratégia do Oceano Azul), 8 'Inovation' (Inovaçao) e 'Value Inovation' (Inovação de Valor), 5 'Strategy' (Estratégia), e 4 'Competitive Strategy' (Estratégia Competitiva), as demais se repetem menos de 4 vezes. Agrupamos as demais palavras de acordo com o seu significado contextualizando em 10 assuntos de maior evidência no conjunto de palavras, e apresentamos na figura 17. 


\begin{tabular}{|l|c|c|}
\hline \multicolumn{1}{|c|}{ Temas } & Quantidade & Percentual \\
\hline Oceano Azul (Blue Ocean) & 57 & $13 \%$ \\
\hline Estratégia (Strategy) & 28 & $6 \%$ \\
\hline Valor (Value) & 24 & $5 \%$ \\
\hline Inovação (Innovation) & 21 & $5 \%$ \\
\hline Negócios (Business) & 18 & $4 \%$ \\
\hline Cliente (Customer) & 15 & $3 \%$ \\
\hline Gestão (Management) & 11 & $3 \%$ \\
\hline Competitividade (Competitive) & 9 & $2 \%$ \\
\hline Produto (Product) & 7 & $2 \%$ \\
\hline Economia (Economic) & 5 & $1 \%$ \\
\hline Outros (Others) & 243 & $55 \%$ \\
\hline
\end{tabular}

Figura 17: Resultados de pesquisa na Scopus - Palavras-chave Agrupadas por Assunto.

As publicações foram escritas por 157 autores, os dois criadores da Teoria Estratégia do Oceano Azul, Kim e Mauborgne apresentam apenas 4 publicações, outros três autores possuem 3 publicações, vinte autores possuem 2 publicações e os demais 132 autores apenas uma publicação, como pode ser observado na figura 18. Os 115 Artigos foram citados 546 vezes em 482 publicações diferentes, onde o índice " $h$ " dos documentos é de valor igual a 10, este índice tem a intenção de quantificar a produção científica a partir das citações dos trabalhos publicados e suas citações (HIRSCH, 2005).

\begin{tabular}{|l|c|}
\hline \multicolumn{1}{|c|}{ Autores } & Artigos \\
\hline Kim, W.C. & 4 \\
\hline Mauborgne, R. & 4 \\
\hline Cascini, G. & 3 \\
\hline Borgianni, Y. & 3 \\
\hline Rotini, F. & 3 \\
\hline 20 Autores & 2 \\
\hline 132 Autores & 1 \\
\hline
\end{tabular}

Figura 18: Resultados de pesquisa na Scopus - Autores com mais de 3 resultados

\section{CONSIDERAÇÕES FINAIS}

A estratégia do oceano azul já possui uma década de existência, seus conceitos estão sendo utilizados em diversas áreas, desde a concepção de desenvolvimento de software (BATISTA et al., 2018), avaliação de um supermercado na cidade de Campina Grande no estado da Paraíba (XAVIER et al., 2015), na melhoria da educação de médicos osteopatas, com o intuito de diferenciar e melhorar a educação tradicional de medicina na busca por melhores médicos osteopatas(SLOCUM et al., 2014), na criação de um modelo de negócios para grupos de comerciantes gregos (BOURLETIDIS, 2014), e até mesmo como direção na busca de análises de mercados juntamente com "Inteligência de Negócios", em inglês “Business Intelligence - Bl" (REBÓN, 2011).

Isso mostra que apesar de recente, a metodologia se provou de fácil utilização em áreas diversas, não sendo restrita ou exclusiva a qualquer tipo de empresa, seja ela pequena, média ou grande (LINDIC et al., 2012). O elevado número de resultados encontrados para as expressões 'Blue Ocean Strategy' e 'Estratégia do Oceano Azul', e a taxa média de crescimento, elevada nos cinco anos após o lançamento do livro, 2.588 resultados, e ainda positiva nos cinco anos posteriores, 4.213 resultados, observado no 
Scholar.google demonstram que o tema permanece relevante, com mais de setecentos resultados abordando o tema somente no ano de 2015.

$\mathrm{Na}$ base Scopus temos mais de cem publicações sobre o assunto, isso demonstra que o tema vem sendo debatido academicamente, com mais de 10 publicações em seis anos do período analisado e com a nova edição do livro que contará com mais três capítulos, certamente o assunto continuará sendo pesquisado e procurado tanto pelas pesquisas acadêmicas como pelo setor empresarial. Apesar do baixo número de artigos encontrados no portal da Anpad o tema ainda possui espaço para pesquisas acadêmicas em português, pois temos apenas $8,4 \% \%$ de resultados em português em comparação com a expressão em língua inglesa considerando apenas 2015, ano em que esta relação foi a maior entre o período analisado, e nos últimos três anos, entre 2013 e 2015 temos mais de 60 resultados, por ano, para a pesquisa em língua portuguesa no website www.scholar.google.com.br.

Em dissertações de mestrado a estratégia do oceano azul vem sendo aplicada como ferramenta de implementação de estratégia (SANTOS, 2011; PATO, 2011; BARROS, 2014; SILVA, 2013) utilizada para comparação com outras estratégias (MARIM, 2010; GUIMARÃES, 2014) e também em desenvolvimento de novos produtos, (KAMPA, 2009). Em tese de doutorado a teoria foi utilizada como referência para implementação de estratégias (BOUER, 2008).

Desta forma o presente artigo atingiu o seu objetivo de mostrar que o tema permanece relevante, tanto quantitativamente, pelos números encontrados, como também qualitativamente, elucidando a relevância do tema com a amostragem realizada da utilização do método criado nas mais diversas áreas de atuação, suas definições estão sendo difundidas em diversas empresas.

As ferramentas apresentadas para a aplicação da teoria estão sendo utilizadas e as empresas estão podendo comprovar se estão no oceano azul ou no oceano vermelho, e o que é melhor, os apontamentos da utilização da metodologia criada estão permitindo que as empresas busquem a inovação de valor para atingir o objetivo de alcançar o crescimento sustentável além da concorrência dos mares vermelhos. Para aplicação de novas pesquisas sugere-se comparar os resultados encontrados sobre a Estratégia do Oceano Azul com outras estratégias, assim como a realização de pesquisas em outras bases.

\section{REFERÊNCIAS}

BARBERO, E. R.; VIEIRA, B. N.. Estratégia do Oceano Azul: Relato de Implantação em um setor em crise. Revista IberoAmericana de Estratégia, p.135-148, 2015.

BARROS, G. A. B.. BOSS: concepção de jogos digitais baseada na estratégia do oceano azul. Dissertação (Mestrado em Ciências da Computação) - Universidade Federal de Pernambuco, Recife, 2014.

BATISTA, G. H. C.; SILVA JUNIOR, I. G.; SOUZA, R. A. C.. Experimentação da Estratégia do Oceano Azul na concepção de produtos de software. Revista Brasileira de

Administração Científica, Aquidabã, v.3, n.2, p.146-162, 2012.

BOUER, R.. Fatores determinantes para a tradução da estratégia em ações e resultados: a disciplina da execução.
Tese (Doutorado em Engenharia) - Escola Politécnica da Universidade de São Paulo, São Paulo, 2008.

BOURLETIDIS, D.. The Strategic Model of Innovation Clusters: Implementation of Blue Ocean Strategy in a typical Greek Region. Procedia - Social and Behavioral Sciences, p.645652, 2014.

CARVALHO, L. S. D.. Aplicação da estratégia do oceano azul em uma empresa de tecnologia da informação no agronegócio: o caso TBIT. Revista Inteligência Competitiva, p.1-17 2014.

CHANG, S. C. Bandit cellphones: A blue ocean strategy. Technology in Society, p.219-223, 2010. 
CRUZ JÚNIOR, J. B. D.; BAUMGARTEN, M. Z.; PEREIRA, M. F.. As melhores práticas de estratégia: o caso da Nintendo CO. Revista da FAE, v.11, n.2, p.1-14 2008.

GALHANONE, R. F.; DECOSTER, S. R. A.. Posicionamento da marca-país Brasil: uma proposta de estratégia do oceano azul. NTERNEXT - Revista Eletrônica de Negócios Internacionais da ESPM, p.120-142, 2007.

GUIMARÃES, C. S. S.. Reconstruir as fronteiras do mercado através de uma estratégia híbrida sustentável. Dissertação (Mestrado em Gestão e Empreendedorismo) - Instituto Politécnico de Lisboa, Lisboa, 2014.

HIRSCH, J. E.. An index to quantify an individual's scientific research output. Washington: PNAS, 2005.

HOLLENSEN, S. The Blue Ocean That Disappeared: the case of Nintendo Wii. Journal of Business Strategy, p.25-35, 2013.

JOÃO, B. N.. Inovação de Valor: O caso Citizenm Hotels. Revista Turismo Visão e Ação, p.299-310, 2011.

JUSSANI, A. C.; KRAKAUER, P. V. D. C.; POLO, E. F. Reflections on Blue Ocean Strategy: a comparison with Ansoff's, Porter's, and Hax and Wilde's strategies. Future Studies Research Journal, p.17-35, 2010.

KAMPA, J. R.. Sistemática para identificação de oportunidades inexploradas de desenvolvimento de novos produtos: Uma proposta baseada na estratégia do oceano azul e no processo de desenvolvimento de novos produtos. Dissertação (Mestrado em Engenharia) - Universidade Tecnológica Federal do Paraná, Curitíba, 2009.

KIM, C.; YANG, K. H.; KIM, J.. A strategy for third-party logistics systems: a case analysis using the blue ocean strategy. Omega The Internacional Journal of Management Science, p.522-534, 2008.

KIM, W. C.; MAUBORGNE, R.. A estratégia do oceano azul: como criar novos mercados e tornar a concorrência irrelevante. Rio de Janeiro: Elsevier, 2005.

LINDIC, J.; BAVDAZ, M.; KOVACIC, H.. Higher growth through the Blue Ocean Strategy: Implications for economic policy. Research Policy, p.928-938, 2012.

MARIM, D.. Estratégias na Indústria de Motocicletas: Um estudo exploratório do setor de motocicletas brasileiro. Dissertação (Mestrado em Administração). Pontifícia Universidade Católica de São Paulo, São Paulo, 2010.

MINTZBERG, H.; AHLSTRAND, B.; LAMPEL, J.. Safári de Estratégia: um roteiro pela selva do planejamento estratégico. Porto Alegre: Bookman, 2000.

OLIVEIRA, D. D. P. R. D.. Planejamento Estratégico: conceitos, metodologia e práticas. São Paulo: Atlas, 2013.

OLIVEIRA, R. T. A. D.. Design de produtos de software dirigidos à inovação: um estudo de caso. Revista Brasileira de Administração Científica - RBADM, Aquidabã, v.4, n.2, p.107-119, 2013.

PATO, A. R.. A academia municipal de golfe de cantanhede e a estratégia oceano azul. Dissertação (Mestrado em Ciências do Desporto) - Faculdade de Ciências do Desporto e Educação Física da Universidade de Coimbra, Coimbra, 2011.

PORTER, M.. Competitive Estrategy: techniques for analyzing industries and competitors. New York: The Free Press, 1980.

PUBLISHNEWS. Oceano de Estratégias. São Paulo: PUBLISHNEWS, 2015.

RANDALL, R. M. W.. Chan Kim and Renée Mauborgne dispel blue ocean myths. Strategy \& Leadership, v.43, n.2, p.11-14, 2015.

REBÓN, F.. Discovering insights within a Blue Ocean based on Business Intelligence. Procedia: Social and Behavioral Sciences, p.66-74,2014.

SANTOS, S. H.. Factores Competitivos: Mergulhando no Turismo de Surf. Dissertação (Mestrado em Marketing) Instituto Universitário de Lisboa, Lisboa, 2011.

SHEEHAN, N. T.; VAIDYANATHAN, G.. Using a value creation compass to discover "Blue Oceans". Strategy \& Leadership, p.13-20, 2009 .

SILVA, M. D. F. B.. O sistema de comunicação como gerador de resultados positivos no IFAM: um estudo de caso no campus manaus distrito industrial. Dissertação (Mestrado em Engenharia de Produção) - Universidade Federal do Amazonas, Manaus, 2013.

SLOCUM, P. C.; PAPA, F.. Envisioning a global role for osteopathic medicine in the 21st century: Using Blue Ocean Strategy to promote osteopathic health care as the World's leader in healthcare education and do it more efficiently, effectively and at the lowest cost. International Journal of Osteopathic Medicine, p.38-42, 2014.

STABELL, C. B.; FJELDSTAD, O. D.. Configuring value for competitive advantage: on chains, shops, and networks. Strategic Management Journal, p.413-437, 1998.

WRIGHT, P. L.; KROLL, M. J.; PARNELL, J.. Administração estratégica: conceitos. São Paulo: Atlas, 2009.

WUBBEN, E. F. M.; DÜSSELDORF, S.; BATTERINK, M. H. Finding uncontested markets for European fruit and vegetables through applying the Blue Ocean Strategy. British Food Journal, v.114, n.2, p. 248-271, 2012.

XAVIER, L. C.; BARBOSA, R. F.; SOUSA, J. E. D.. A estratégia do oceano azul: um estudo de caso numa empresa de serviços na cidade de campina grande - PB. Geintec - Gestão, Inovação de Tecnologias, p.2348-2365, 2015.

YANG, J. T.. Identifying the attributes of blue ocean strategies in hospitality. International Journal of Contemporary Hospitality Management, p.701-720, 2012. 